Gefässchirurgie 2019 · 24:592-593

https://doi.org/10.1007/s00772-019-00587-2

(c) Springer Medizin Verlag $\mathrm{GmbH}$, ein Teil von Springer Nature 2019

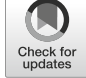

\section{F. Adili' · A. Larena-Avellaneda ${ }^{2}$}

'Klinik für Gefäßmedizin - Gefäß- und Endovascularchirurgie, Klinikum Darmstadt, Darmstadt, Deutschland

${ }^{2}$ Klinik und Poliklinik für Gefäßmedizin, Universitäres Herzzentrum Hamburg, Universitätsklinikum Hamburg-Eppendorf, Hamburg, Deutschland

\title{
Neue Lehre braucht das Land
}

Geschätzte Leserinnen und Leser,

es ist wieder soweit. Heft 8 ist im Entstehen begriffen, und wenn Sie es lesen, neigt sich das Jahr dem Ende zu. Das Editorial entsteht allerdings deutlich früher, auch wenn es in den Läden schon reichlich Weihnachtsutensilien zu kaufen gibt. Näher liegt noch der Nikolaustag. Ganz oberflächlich betrachtet, haben wir es mit einem Erziehungsprinzip zu tun. Der 06.12. verdankt seinen Namen dem Bischof von Myra, er gilt als Todestag des heiligen Nikolaus. Der Legende nach fehlte einem armen Mann das Geld für die Heirat seiner 3 Töchter. Als Nikolaus davon erfuhr, warf er Gold in den Kamin der Familie. Dieses fand sich dann in den Stiefeln und Socken wieder. Daher soll der heilige Nikolaus jedes Jahr durch den Kamin kommen und artigen Kindern Geschenke in die Schuhe oder Strümpfe legen. Also ein gutes Belohnungsprinzip, wobei es hier durchaus auch gegenteilige Meinungen beim Einsatz von Belohnungen gibt [1]. Während man aber nichts an der Schule ändern kann, ist die Teilnahem am Medizinstudium (meist) freiwillig. Und hier kommt die Motivation ins Spiel. Es gibt etwa 9400 Studienplätze für Humanmedizin, aber viermal so viele Bewerber. Warum wollen so viele junge Menschen Medizin studieren?

\section{》) Es gibt etwa 9400 Studien- plätze für Humanmedizin, aber viermal so viele Bewerber}

Es folgen weitere Fragen - wie kann man die Studierenden begeistern, motivieren, Inhalte dauerhaft beibringen? Viel hat sich hier seit unseren Studientagen getan.
Die Digitalisierung tut ihr übriges, dass Lehrveranstaltung heute anders funktionieren als noch vor 20 Jahren. Der Lehrende steht dabei viel mehr als früher im Fokus, und an fast jeder Universität wird die Lehre evaluiert. Dementsprechend werden die Dozentinnen und Dozenten regelhaft geschult, wie auch die Umfrage in dieser Ausgabe bestätigt.

Eine grundsätzliche Arbeitsweise vieler Didaktik-Workshops, inklusive unserer Train-the-Trainer-Kurse, die jeder wissenschaftliche Leiter eines DGG-akkreditierten Fort- und Weiterbildungskurses absolviert haben muss, ist das sog. „Box-in-the-box"-Prinzip. D. h. Lehrmethoden, die in den Didaktikkursen verwendet werden, sollen von den Teilnehmern der Kurse zunächst persönlich erlebt und später bei den eigenen Lehrveranstaltungen und -formaten möglichst 1:1 umgesetzt werden. Lassen Sie uns dies für die Einführung in das hier vorliegende Leitthemenheft „Lehre“ einmal praktisch demonstrieren:

"If you can't explain it simply, you don't understand it well enough. "Dieses Zitat, das Albert Einstein zugeschrieben wird, birgt viel Wahrheit. Wer erinnert sich nicht an Bilder, Tipps, Tricks, Metaphern oder „Chirurgen-Weisheiten“, mit denen uns unsere früheren Lehrer konfrontiert haben, bzw. derer wir uns selbst bedienen und die von Generation zu Generation tradiert werden. Komplexe Sachverhalte oder Skills werden so dargestellt, erläutert und gelehrt, dass selbst „die eigene Großmutter" sie verstehen und umsetzen könnte. „Kinderleicht“ eben. Dies funktioniert natürlich dort am besten, wo Lehrende ihr Fach exzellent beherrschen.
Hängt eine wirksame Lehre jedoch wirklich nur von der Fachlichkeit, Erfahrung und Seniorität des Lehrenden ab? Oder anders formuliert: Kann ein „Meisterchirurg" tatsächlich jedem „Affen“ das Operieren beibringen? Wohl kaum.

Die moderne Didaktik lehrt uns, dass Kompetenzen nicht nach dem Gießkannenprinzip für alle gleichartig verbreitet, sondern nach den unterschiedlichen Bedürfnissen der Lernenden individualisiert, vermittelt werden sollten, um besser wirksam zu werden. Am besten gelingt das dort, wo bei Lehrenden Wissen, Können, Erfahrung, die Bereitschaft diese zu teilen und eine gewisse "Passion" für die Lehre zusammenkommen. Für den Lernenden andererseits, führt ein positives Lernklima, welches die Selbstwirksamkeit, das Autonomieerleben, einen wahrnehmbaren Kompetenzzuwachs und ein Gefühl der Akzeptanz und Wertschätzung fördert, zu einer Steigerung der Motivation zu lernen, zu handeln und sich etwas zuzutrauen. Für das Herstellen dieses Lernklimas sind die Lehrenden maßgeblich verantwortlich. Damit Lernen individualisiert erfolgen kann, muss der individuelle Lernfortschritt regelmäßig erfasst, gemessen, geprüft werden. Und jetzt kommen wir zum „Box-in-the-box“-Prinzip zurück, nämlich zu der folgenden Metapher: "Teaching (training) without testing, is like cooking without tasting."

\section{》) Für das Herstellen eines positiven Lernklimas sind die Lehrenden verantwortlich}

Die novellierte Musterweiterbildungsordnung, die spätestens im nächsten Jahr 
auch für uns Gefäßchirurgen in Deutschland flächendeckend umgesetzt werden soll, basiert darauf, dass nicht allein bestimmte Rotationen, Mindestzeiten und Mindestzahlen von Prozeduren absolviert sein müssen, bevor man sich zum abschließenden „kollegialen Gespräch“ anmelden darf, sondern das Erreichen einer geforderten Mindestkompetenz, die sog. Facharztreife. Erst wenn der/die Absolvent/in wirklich "kompetent" geworden ist und dies auch gezeigt hat, darf er/sie sich zur Facharztprüfung anmelden, die übrigens diesen Namen, zumindest in Deutschland, kaum verdient. Warum nicht? Weil durch eine mündliche Befragung nicht festgestellt werden kann, ob ein Kandidat in einem klinischen Szenario tatsächlich so performen kann, wie er/sie im Gespräch behauptet. Dies gilt in besonderem Maße für operative Fächer. Hierzu bedarf es einer praktischen Prüfung, ähnlich der Fahrprüfung beim Führerscheinerwerb. Noch so ein vergleichendes Bild ...

Die unterschiedliche Wirksamkeit verschiedener Lehr- und Prüfungsmethoden, lässt sich sowohl mit Methoden der biomedizinischen Forschung als auch den uns weniger vertrauten $\mathrm{Me}$ thoden der Sozialwissenschaften und Psychologie exzellent beforschen und hochklassig publizieren. Mehr noch, Exzellenz in der Lehre lässt sich überhaupt nur durch gleichzeitiges Forschen und Publizieren erreichen. Gut geplante und durchgeführte Studien, die zu einer evidenzbasierten Lehre (Best Evidence Medical Education - BEME) führen, werden regelmäßig im NEJM, Lancet, JAMA oder BMJ publiziert. Die klassischen "Lehr-Journale“ wie Academic Medicine oder Medical Education haben einen Impact-Faktor von 4,8 bzw. 4,6 und übertreffen damit sämtliche gefäßchirurgische Zeitschriften. Lehrforschung $\mathrm{zu}$ betreiben und $\mathrm{zu}$ publizieren kann also einer akademischen Laufbahn sehr dienlich sein. In universitären, aber auch chefärztlichen Berufungsverhandlungen spielen die Lehrexpertise bzw. Fragen zur Lehrbefähigung heutzutage eine immer größere Rolle.

Die in dieser Ausgabe der Gefässchirurgie publizierten Arbeiten geben einen Eindruck darüber, was eine Professiona- lisierung der Lehre für den Unterricht abseits und im klinischen Alltag bedeuten kann und mögen Anregung sein, sich intensiver mit Fragen der Lehre auseinanderzusetzen und sich selbst medizindidaktisch (weiter) zu professionalisieren.

Exzellente Lehre führt $\mathrm{zu}$ besseren Ärztinnen und Ärzten. Sie untermauert den akademischen Anspruch unseres Fachs. Nicht nur wird gefäßchirurgisches Wissen an alle Studierenden vermittelt, sondern eine exzellente Lehre sichert damit unsere Zukunft als eigenständige Disziplin und die Weiterbildung zu kompeteten Gefäßchirurgen. Sie verhilft uns zu größerer Attraktivität in den Augen des Nachwuchses und stärkt unsere Innovationskraft. In diesem Sinne wünschen wir Ihnen interessante Einsichten, Anregungen und viel Spaß bei der Lektüre.

Ihre

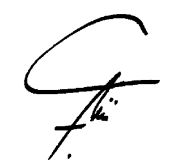

PD Dr. F. Adili

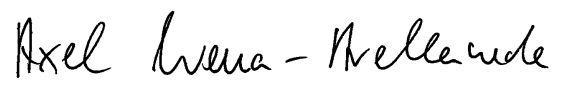

Prof. Dr. A. Larena-Avellaneda

\section{Korrespondenzadresse}

PD Dr. med. F. Adili
Klinik für Gefäßmedizin
- Gefäß- und Endovas-
cularchirurgie, Klinikum
Darmstadt
Grafenstr.9,64283 Darmstadt,
Deutschland
farzin.adili@
mail.klinikum-darmstadt.de

Interessenkonflikt. F. Adili und A. Larena-Avellaneda geben an, dass kein Interessenkonflikt besteht.

\section{Literatur}

1. Dietz H (2017) Belohnen ist das neue Bestrafen. https://www.zeit.de/kultur/2017-03/ erziehung-belohnungen-psychologie-verhaltenmotivation-10nach8/seite-2. Zugegriffen: 09.10.2019 